\title{
Deficiencia de zinc y sus implicaciones funcionales
}

Jorge L. Rosado, M.Sc., Ph.D.(1)

\section{Rosado JL. \\ Deficiencia de zinc y sus implicaciones funcionales. Salud Publica Mex 1998;40:181-188.}

\section{Resumen}

El presente trabajo tiene por objeto revisar los aspectos teóricos y los estudios realizados en México que sugieren la existencia de la deficiencia moderada de zinc en niños de población rural, así como algunas de las consecuencias de dicha deficiencia en la salud. El zinc es un nutrimento indispensable para el organismo de los humanos y juega un papel importante en una serie de procesos metabólicos: participa en el sitio catalítico de varios sistemas enzimáticos; participa como ion estructural en membranas biológicas, y guarda una estrecha relación con la síntesis de proteínas, entre otras cosas. Es por esto que la deficiencia de zinc está asociada con consecuencias importantes en la salud y la funcionalidad de los individuos, especialmente durante las primeras etapas de la vida. De relevancia para México es la existencia de una deficiencia moderada de zinc en los niños y las consecuencias que ésta pueda tener en la salud de los mismos. Los estudios realizados sugieren que la deficiencia moderada de zinc se presenta asociada con la ingestión de dietas basadas en alimentos de origen vegetal, las cuales contienen cantidades importantes de inhibidores de la absorción de zinc. Este tipo de dietas se consume habitualmente en las zonas rurales y en la po blación marginal de las ciudades en el país. Entre las consecuencias más importantes de esta deficiencia se encontró un aumento en la presencia de enfermedades infecciosas, especialmente de diarrea, y posibles alteraciones en el desarrollo de la capacidad cognoscitiva.

Palabras clave: cinc; disponibilidad biológica; diarrea; enfermedades respiratorias; desarrollo infantil; literatura de revisión; México

\author{
Rosado JL. \\ Zinc deficiency \\ and its functional implications. \\ Salud Publica Mex 1998;40:181-188.
}

\begin{abstract}
A bstract
The purpose of this article is to review theoretical aspects and research performed in Mexico suggesting the existence of marginal zinc deficiency in rural children and its consequences on health. Zinc is an indispensable nutrient for humans since it plays an important role in several metabolic pathways: it participates in the catalytic site of several enzymes, as a structural ion of biological membranes and is closely associated to protein synthesis, among other functions. It is therefore related to functional and health impairments, specially in children. A marginal deficiency of zinc in Mexican children is relevant because of its potential effects. Studies suggest that marginal zinc deficiency is associated to diets based on plant foods wich are rich in zinc absorption inhibitors. Such diets are habitually consumed in rural areas and in poors areas of the cities. Marginal zinc deficiency was found to cause an increase in infectious diseases, specially diarrhea, and an impairment of the cognitive functions.
\end{abstract}

Key words: zinc; biological availability; diarrhea; respiratory tract diseases; child develo pment; review literature; Mexico

(1) Departamento de Fisiología de la N utrición, Instituto N acional de la N utrición Salvador Zubirán, México.

Fecha de recibido: 10 de julio de 1997 - Fecha de aprobado: 23 de enero de 1998 


\section{Metabolismo y esencialidad del zinc}

E 1 zinc posee una serie de propiedades químiE cas que lo hacen único y muy útil en varios sistemas biológicos y, por lo tanto, partícipe de un gran número de procesos metabólicos. ${ }^{1}$ Tiene un papel muy activo en el sitio catalítico de un número importante de sistemas enzimáticos; a diferencia del hierro y del cobre, no cambia su estado electroquímico, por lo que no es útil en reacciones de óxido-reducción; sin embargo, por la misma razón, el organismo no corre riesgo de daño por oxidación, lo que permite que el zinc sea transportado y utilizado mas fácilmente. Además de su papel como ion catalítico, el zinc también destaca por ser un ion estructural que participa en algunas membranas biológicas o en los ácidos nucleicos. ${ }^{2,3}$ Puede formar enlaces cruzados como en las bases de los llamados "dedos de zinc", que caracterizan a algunas proteínas de transcripción. ${ }^{4,5}$ El zinc es necesario para la integridad de las histonas, proteínas íntimamente involucradas con el ADN, además de ser un componente de las polimerasas del ADN y del ARN y de enzimas citosólicas involucradas en la síntesis de proteínas, razón por la cual se ha mencionado que el zinc puede desempeñar un papel central en el crecimiento celular. ${ }^{1}$ Por otra parte, también se ha sugerido que el zinc puede ser un regulador intracelular con una importancia biológica similar a la del calcio; sin embargo, es poco lo que se sabe acerca de esa función. ${ }^{3}$

En consideración a la extraordinaria variedad de funciones biológicas de este nutrimento, hasta el momento ha resultado prácticamente imposible asociar la bioquímica del zinc con los aspectos clínicos y funcionales debidos a su deficiencia. Sin embargo, es claro que el aporte de este nutrimento por debajo de las cantidades apropiadas puede interferir con la función celular en gran variedad de sitios.

\section{Deficiencia de zinc en la población mexicana}

Las deficiencias de cualquier nutrimento pueden deberse a una disminución en la ingestión del mismo, a un aumento en las necesidades del organismo y, por lo tanto, en sus requerimientos, o bien, a una disminución en la biodisponibilidad del nutrimento por la forma en que éste se encuentra en los alimentos.

Los estudios en los que se ha fundamentado el diagnóstico de la situación nutricia de vitaminas y minerales en México se han elaborado a partir de datos procedentes de encuestas de dieta que, al combinarse con la información del contenido de nutrimentos de los alimentos (tablas), arrojan el valor de consumo de algún nutrimento en particular. En el caso del zinc no se ha evaluado su nivel de ingestión debido, probablemente, a dos razones: la primera, que aún no se reconoce su importancia nutricia, a pesar de su destacado papel bioquímico, estructural y regulador; la segunda es que no se dispone de información acerca del contenido de zinc en los alimentos que se consumen en México. Y, ciertamente, aunque existiera la información de niveles de ingestión, ésta no es sinónimo del estado de nutrición, razón por la cual debe interpretarse con mucha precaución. En algunos casos, como en el del hierro, esos datos son irrelevantes, y quizá para el zinc la situación es muy similar.

En cuanto a la evaluación bioquímica del estado nutricio de zinc en la población mexicana, existen únicamente dos estudios publicados. Hunt y colaboradores $^{6}$ realizaron una serie de mediciones bioquímicas, además de un recordatorio de la dieta de 24 horas en 66 mujeres embarazadas atendidas en diferentes clínicas en Montemorelos, Nuevo León: la ingestión promedio de zinc en el embarazo fue baja $(7.8+3.3 \mathrm{mg} / \mathrm{d})$, y $57 \%$ de las mujeres presentaron valores bajos de zinc en plasma $(<8.1 \mathrm{umol} / \mathrm{l})$. Rosado y colaboradores $^{7}$ estudiaron indicadores bioquímicos asociados con el estado nutricio del zinc en 219 prescolares de una zona rural del Estado de México; 20\% de los niños presentaron concentraciones bajas de zinc en plasma $(<10.7 \mathrm{umol} / \mathrm{l})$. Estos hallazgos sugieren deficiencia de zinc en algunos grupos del país, lo cual amerita una identificación más clara.

\section{Biodisponibilidad del zinc en la dieta mexicana}

Como ya se mencionó, la deficiencia de nutrimentos puede deberse, entre otras causas, a cambios en la absorción y la utilización de los mismos. En el caso específico del zinc, el intestino delgado mantiene básicamente su homeostasis; en circunstancias normales el aparato gastrointestinal se adapta a los cambios en la ingestión o en el estado nutricio de zinc produciendo cambios en la porción del nutrimento que se absorbe o en la excreción fecal de zinc endógeno. ${ }^{8}$ Inclusive una cantidad importante de este último que se secreta al lumen del tracto gastrointestinal se reabsorbe continuamente para evitar un balance negativo de zinc. Cualquier cambio que modifique la fisiología normal del tracto digestivo afecta profundamente la homeostasis del zinc. ${ }^{9}$

El estado nutricio del zinc depende de la cantidad ingerida y de su biodisponibilidad. Los alimentos de origen animal, particularmente las carnes, los mariscos y el pescado, son fuentes ricas en zinc, y su biodispo- 
nibilidad es alta ya que durante su digestión se liberan ciertos aminoácidos y péptidos que contienen lisina y que forman complejos solubles con el zinc. ${ }^{10}$ En México la contribución de los alimentos de origen animal a la dieta es muy limitada, en particular en la población rural y en la marginal urbana, ${ }^{11}$ cuya dieta se restringe al maíz y al frijol, adicionados con cantidades variables de verduras y frutas. Estas dietas presentan concentraciones elevadas de ácido fítico y de fibra dietética que inhiben la absorción de zinc. El ácido fítico es la forma principal en la que se almacena el fósforo en los cereales, las leguminosas y las oleaginosas y es el inhibidor más potente de la absorción de zinc, ${ }^{12}$ ya que forma complejos insolubles a $\mathrm{pH}$ fisiológico. En relación con las fibras dietéticas, se ha sugerido que las insolubles, como la celulosa y la lignina -que se encuentran sobre todo en los cereales-, contribuyen a disminuir la absorción del zinc. ${ }^{13}$ Otro factor que puede afectar su absorción y que es relevante en las dietas que se consumen en México es el calcio, cuyos niveles elevados en la dieta pueden formar complejos de Ca:Zn:fitatos que son menos solubles que los complejos formados por los minerales solos. ${ }^{14}$

En el cuadro I se muestra el consumo de zinc y de sus principales inhibidores en modelos de dietas típicas tanto rurales como urbanas. ${ }^{15,16}$ Puede observarse que a pesar de que no hay una gran diferencia en el contenido de zinc entre ambos modelos de dieta, la rural contiene cuatro veces más fibra dietética, 18 veces más ácido fítico y 1.8 veces más calcio; este último proviene de la adición de hidróxido de calcio durante la nixtamalización de la tortilla. Así, es de esperar que la dieta rural típica constituya un factor que disminuya la absorción de zinc y un factor potencial de riesgo para la existencia de deficiencia moderada de zinc en la población del país.

Con el objeto de evaluar la absorción de zinc en las dietas que se consumen en México, Rosado y colaboradores ${ }^{16}$ diseñaron modelos de dietas rural y urbana, ambas representantes del consumo habitual de alimentos en las zonas respectivas del país de acuerdo con los resultados de las encuestas nacionales de alimentación. ${ }^{17}$ Con ambas dietas se determinó el contenido, la absorción y la utilización de zinc mediante estudios de balance realizados en 16 mujeres en edad reproductiva. Como puede observarse en la figura 1, la ingestión de zinc fue muy similar con ambas dietas; sin embargo, $96 \%$ del zinc ingerido se excreta en las heces con la dieta rural, mientras que con la urbana ese valor es de $84 \%$. La cantidad absorbida de zinc fue tres veces mayor con la dieta urbana que con la rural, y el balance fue doce veces mayor. Estos resultados demuestran que la dieta que se consume normalmente

\section{Cuadro I \\ INGESTIÓN DE ZINC Y DE INHIBIDORES \\ DE SU ABSORCIÓN EN MODELOS DE DIETAS RURALES Y URBANAS MEXICANAS*}

\begin{tabular}{lll} 
Componente & Dieta rural & Dieta urbana \\
Zinc $(\mathrm{mg} / \mathrm{d})$ & $10.9 \pm 1.0$ & $10.0 \pm 1.9$ \\
$\begin{array}{c}\text { Acido fítico } \\
(\mathrm{mmol} / \mathrm{d})\end{array}$ & $1.62 \pm 0.14$ & $0.09 \pm 0.02$ \\
\hline$(\mathrm{g} / \mathrm{d})$ & $1.07 \pm 0.09$ & $0.07 \pm 0.01$
\end{tabular}

Fibra dietética $(\mathrm{g} / \mathrm{d})$

\begin{tabular}{lrr} 
N eutro detergente & $40.2 \pm 2.6$ & $12.1 \pm 0.9$ \\
\hline A cido detergente & $28.7 \pm 1.8$ & $8.2 \pm 0.5$ \\
\hline Hemicelulosa & $11.4 \pm 1.6$ & $3.7 \pm 1.0$ \\
\hline Celulosa & $17.4 \pm 1.4$ & $5.5 \pm 0.7$ \\
\hline Lignina & $11.3 \pm 1.3$ & $2.6 \pm 0.2$
\end{tabular}

Calcio (mg/d) $\quad 745 \pm 70 \quad 410 \pm 30$

* Modificado de Mukherjee et al. y Cherry y et al. 58,59

en las zonas rurales de México ejerce un efecto negativo importante en la biodisponibilidad del zinc, y que esta disminución en la absorción y en la retención del nutrimento puede ser un mecanismo que ocasione deficiencia de zinc en una porción importante de la población nacional.

\section{Consecuencias fisiológicas y funcionales de la deficiencia de zinc}

La deficiencia de zinc en humanos se notificó por primera vez en Egipto ${ }^{18}$ durante los años sesenta. El fenómeno se presentaba en adolescentes del sexo masculino con enanismo nutricio yse caracterizaba por un retraso muy importante en el crecimiento y en la maduración sexual. Posteriormente se demostró que los afectados respondían favorablemente al tratamiento con zinc. ${ }^{19,20}$

En la actualidad los casos de deficiencia severa de zinc son más bien raros; sin embargo, la deficiencia moderada ha sido ampliamente notificada. Los grupos de población más afectados por la deficiencia de zinc son los niños menores y las mujeres durante el embarazo y la lactancia. La mayoría de los estudios realizados en niños, tanto de países avanzados ${ }^{21-29}$ como de naciones en desarrollo, ${ }^{30-43}$ no notificaron signos aparentes de deficiencia clínica de zinc; en cambio, sí indicaron deficiencia moderada del mineral caracterizada por retraso en el crecimiento lineal; efecto negativo en la sensibilidad sensorial, especialmente 


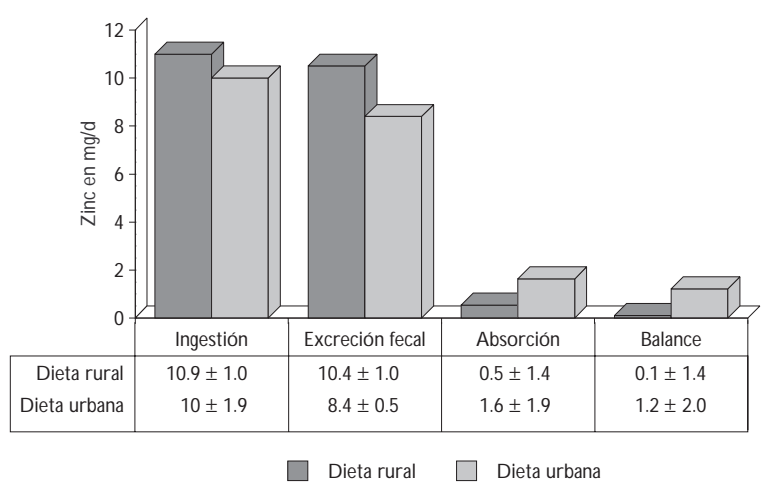

Figura 1. INGESTIÓN, EXCRECIÓN FECAL, ABSORCión APARENTE Y BALANCE DE ZINC OBTENIDO POR 16 MUJERES EN ESTUDIOS DE BALANCE METABÓLICO CON MODELOS DE DIETA RURAL Y URBANA. LAS DIFERENCIAS EN LA EXCRECIÓN FECAL Y EN LA ABSORCIÓN SON ESTADÍSTICAMENTE SIGNIFICATIVAS $(P<0.05)$

en la capacidad para detectar sabor; disminución en el apetito; disminución en la respuesta inmune y efectos negativos en la función intestinal, que constituyen los padecimientos más comunes en el individuo con deficiencia de zinc.

Cabe destacar que la evidencia clara de deficiencia de zinc determinada por indicadores bioquímicos no ha sido un hallazgo consistente en la mayoría de los estudios publicados. Lo anterior se explica porque las consecuencias funcionales de la deficiencia de zinc aparecen antes de que los niveles en plasma y/o en algún otro tejido se vean disminuidos. ${ }^{29,44}$ Esto pone de relieve la importancia de confirmar la deficiencia moderada de zinc mediante la respuesta positiva a la suplementación en estudios doble ciego.

En los niños con deficiencia de zinc pueden presentarse diversas adaptaciones que contribuyen a cumplir con el incremento en las necesidades de zinc, y que incluyen un aumento en la capacidad de absorción intestinal, una disminución en las pérdidas endógenas o la redistribución de zinc en los tejidos. Si bien estos mecanismos pueden bastar para disminuir el riesgo de deficiencia del nutrimento en un gran número de niños, otros factores como el deterioro en el estado nutricio de zinc debido a la presencia de infecciones, el aumento en las pérdidas endógenas del mineral o su baja absorción debido a la ingestión de dietas con baja biodisponibilidad, pueden favorecer la presencia de deficiencia moderada de zinc. Lamentablemente existen muy pocos estudios en los que se haya evaluado el estado de nutrición de zinc en poblaciones con alto riesgo $0^{45,46} \mathrm{y}$, aun menos, estudios que hayan incluido suplementación.

La deficiencia de zinc puede presentarse desde antes del nacimiento de un niño; estudios en animales han demostrado el efecto teratogénico de la deficiencia de zinc. ${ }^{47}$ Sin embargo, los estudios en humanos han sido inconsistentes; en el caso de deficiencia clínica de zinc, como en la acrodermatitis entero hepática, se pueden presentar alteraciones congénitas (p.e., anencefalia) y abortos. ${ }^{48} \mathrm{La}$ deficiencia marginal de zinc durante el embarazo y su efecto en el recién nacido no son tan claros, en parte debido a que, como ya se mencionó, es difícil establecer la existencia de una deficiencia moderada de zinc mediante indicadores bioquímicos. El nivel de zinc en plasma ha sido el indicador más común de deficiencia de zinc en el embarazo; no obstante, este indicador puede disminuir aun en presencia de un estado adecuado de nutrición en la madre ${ }_{1}^{49}$ debido a una expansión del volumen plasmático durante el embarazo. En la mayoría de los estados se han encontrado correlaciones entre el zinc en plasma con una serie de complicaciones durante el embarazo, entre las que destacan un trabajo de parto más prolongado, hipertensión, hemorragias después del parto, abortos y malformaciones congénitas; ;0-56 son pocos los estudios que no han encontrado tal asociación. ${ }^{57,58}$ En estudios doble ciego de suplementación con zinc se ha encontrado una disminución en las complicaciones asociadas con el embarazo en el grupo al que se le administró el suplemento en comparación con el grupo placebo..$^{59,60} \mathrm{En}$ un estudio reciente Goldenberg y colaboradores ${ }^{61}$ encontraron un incremento en el peso al nacer y en la circunferencia cefálica en los niños de mujeres de origen africano en Estados Unidos de América que recibieron $25 \mathrm{mg}$ de zinc al día.

\section{Deficiencia de zinc y crecimiento}

Una de las evidencias más claras de deterioro en el estado nutricio de los niños en México es la alta prevalencia de retraso en el crecimiento, especialmente de un crecimiento lineal, que se muestra como un retraso en la talla para la edad. ${ }^{11}$ Con la finalidad de evaluar si la deficiencia de zinc podría desempeñar un papel etiológico importante en este fenómeno, se llevó a cabo un estudio de suplementación en 219 niños de entre 12 y 36 meses de edad que vivían en una comunidad rural del Estado de México. ${ }^{41}$ La suplementación consistió en la administración diaria, durante un año, de $20 \mathrm{mg}$ de zinc metionina, cuya biodisponibilidad más elevada había sido probada. ${ }^{62}$ El efecto de la suplementación con zinc en comparación con el placebo se muestra en el cuadro II; como puede observarse, no se encontró 
un cambio significativo en ninguno de los indicadores antropométricos.

La falta de efecto de la suplementación con zinc en una población en la que se podía anticipar una alta incidencia de deficiencia moderada de ese nutrimento se debe a varios factores. A pesar de que la incidencia de retraso en el crecimiento dentro del grupo estudiado era importante, no era tan alta como en otras poblaciones donde la suplementación con zinc ha tenido un efecto positivo en el crecimiento lineal; al inicio del estudio los niños presentaban en promedio un retraso en la talla para la edad de -1.6 desviaciones estándar por debajo de la norma internacional.

Una revisión reciente de los estudios que han observado el impacto de la suplementación con zinc en el crecimiento ${ }^{63}$ demuestra que el efecto positivo de la suplementación con zinc en el crecimiento es más evidente cuando el retraso en el crecimiento es mayor y/o cuando los niveles de zinc en plasma están muy bajos. En el estudio de México, 25\% de los niños presentaban valores de zinc en plasma $<70 \mathrm{ug} / \mathrm{dl}$, lo cual se considera como criterio para marcar la deficiencia del mineral. Por otro lado, más de $80 \%$ de los niños del estudio presentaban deficiencia de, por lo menos, dos micronutrimentos, y las deficiencias más comunes fueron la de hierro, riboflavina, vitamina B12, vitamina $\mathrm{E}$, zinc y vitamina $\mathrm{A}$. El retraso en el crecimiento de los niños en México puede ser la resultante más bien del efecto de la deficiencia moderada de varios nutrimentos, por lo que la suplementación con sólo uno de ellos, como es el caso del zinc, tiene un efecto muy marginal. Lo anterior está sustentado por un estudio reciente llevado a cabo en una zona rural del estado de Morelos en donde se suplementó a un grupo de niños con una mezcla de varios micronutrimentos que incluía

\section{Cuadro II \\ Cambio en los valores de peso y talla de los niños EN UNa zona RURAL de MÉxico deSPUÉs de UN año DE SUPLEMENTACIÓN CON ZINC O PLACEBO*}

\begin{tabular}{lrr} 
Parámetros antropométricos & \multicolumn{2}{c}{ Grupos } \\
\cline { 2 - 3 } & \multicolumn{2}{c}{ Placebo } \\
Talla $(\mathrm{cm})$ & $9.1 \pm 0.22$ & $9.3 \pm 0.21$ \\
\hline Peso $(\mathrm{kg})$ & $2.4 \pm 0.07$ & $2.4 \pm 0.07$ \\
\hline$Z$ talla/edad & $0.13 \pm 0.06$ & $0.16 \pm 0.05$ \\
\hline$Z$ peso/edad & $0.25 \pm 0.05$ & $0.26 \pm 0.05$ \\
\hline$Z$ peso/talla & $0.29 \pm 0.06$ & $0.25 \pm 0.06$
\end{tabular}

Nota: $Z$ talla/edad corresponde a puntaje $Z$ de talla para la edad; $Z$ peso/ edad, a puntaje $Z$ del peso para la edad, y $Z$ peso/talla, al puntaje $Z$ del peso para la talla

* Modificado de Gibson et al. ${ }^{29}$ zinc. Rivera y colaboradores ${ }^{64}$ encontraron en dicho estudio que los niños del grupo que recibió suplemento crecieron entre 0.7 y 1.2 centímetros más que los niños del grupo que recibió placebo después de seis meses de suplementación, diferencia que resultó altamente significativa. Estos estudios sugieren que, si bien una de las consecuencias de la deficiencia de zinc es el retraso en el crecimiento, en México este último se debe más bien a un deterioro en el estado de nutrición respecto a varios nutrimentos, entre ellos el zinc, el cual puede desempeñar un papel importante pero no único.

\section{Deficiencia de zinc y morbilidad por enfermedades infecciosas}

La presencia de diarrea afecta el estado de zinc mediante una disminución en la ingestión, cambios en la absorción intestinal y un incremento en las pérdidas en el intestino, ${ }^{65}$ por lo que durante la diarrea aumentan las necesidades de zinc para mantener un balance positivo. ${ }^{65,66}$ De esta manera, en los niños con alta incidencia de diarrea se presenta un ciclo de deprivación de zinc en el que se inicia un balance negativo del nutrimento que aumenta la incidencia de diarrea; esto, a su vez, produce un mayor deterioro en el estado nutricio de zinc, lo que incrementa la diarrea y la desnutrición.

Con el objeto de conocer si la deficiencia de zinc pudiera afectar la presencia de enfermedades infecciosas en la población infantil mexicana se evaluó el efecto de la suplementación con $20 \mathrm{mg} / \mathrm{d}$ de zinc durante un año, en niños de una comunidad rural del Estado de México, que presentaban diarrea, enfermedades respiratorias y otro tipo de padecimientos. ${ }^{41} \mathrm{La}$ figura 2 muestra el efecto de la suplementación con zinc en presencia de episodios totales de enfermedad en la población estudiada. En el grupo al cual se le administró zinc se encontró una disminución de $17 \%$ en la presentación de episodios de enfermedad en comparación con el grupo placebo; el efecto fue marginal en cuanto a enfermedades respiratorias, pues se encontró una reducción de $9 \%$, pero fue más evidente en la presencia de padecimientos diarreicos, ya que la administración de zinc causó una disminución de 35\% en los mismos. En otro tipo de padecimientos, el efecto de la suplementación con zinc fue una reducción de $42 \%$. La disminución de enfermedades diarreicas en el grupo que recibió suplemento con zinc resultó altamente significativa. En contraste, la suplementación con zinc causó una reducción en los episodios de enfermedad, especialmente en el caso de los padecimientos diarreicos, pero no afectó la duración de los mismos (figura 3). 


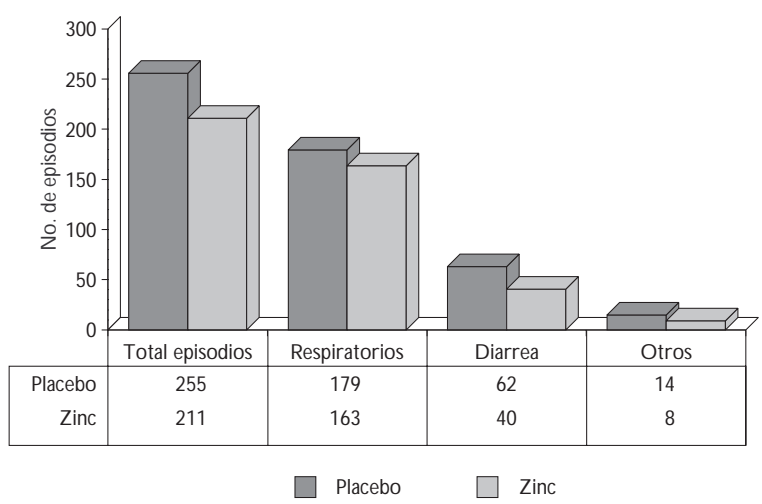

Figura 2. Presencia de enfermedad en niños que RECIBIERON SUPLEMENTO CON ZINC DURANTE 12 MESES $Y$ EN NIÑOS QUE RECIBIERON PLACEBO EN EL MISMO PERIODO. LAS DIFERENCIAS EN LA PRESENCIA DE EPISODIOS DE DIARREA SON ESTADÍSTICAMENTE SIGNIFICATIVAS $(P<0.05)$

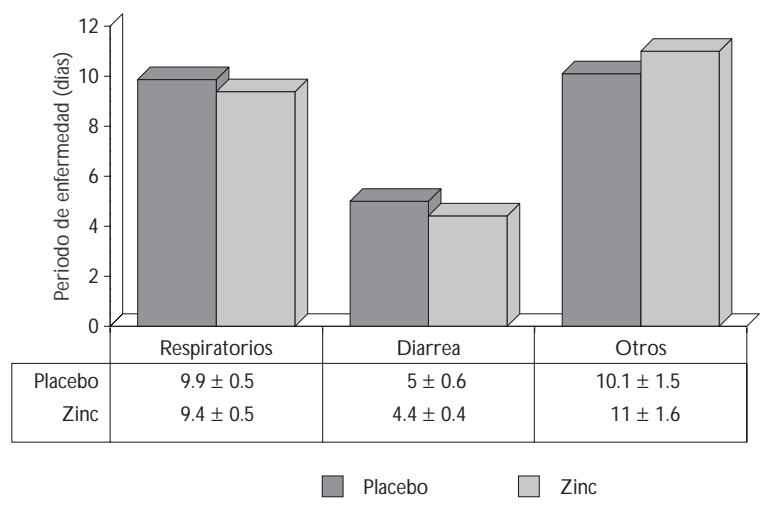

Figura 3. Duración de los periodos de enfermedad EN NIÑOS QUE RECIBIERON SUPLEMENTO CON ZINC DURANTE 12 MESES Y EN NIÑOS QUE RECIBIERON PLACEBO EN EL MISMO PERIOdO. LoS VALORES ENTRE LOS TRATAMIENTOS NO SON ESTADÍSTICAMENTE DIFERENTES

\section{La deficiencia de zinc y el desarrollo cognoscitivo}

El papel del zinc en el desarrollo y las funciones del cerebro ha sido estudiado básicamente en animales. La deficiencia de zinc retrasa el crecimiento y la maduración de las neuronas, y afecta la actividad electrofisiológica y de transmisión en el cerebro mediante mecanismos que no han sido plenamente identificados; ${ }^{67,68}$ el efecto que ello produce en la capacidad cognoscitiva sólo ha sido sugerido en estudios muy preliminares en China. ${ }^{*}$ Para saber si tal efecto existía en niños mexicanos se aplicaron pruebas de desarrollo cognoscitivo a 219 niños de entre 30 y 48 meses de edad, de una zona rural del Estado de México, y las calificaciones obtenidas se asociaron con diferentes indicadores del estado nutricio, incluyendo el zinc. ${ }^{69}$ Los resultados de las pruebas de desarrollo cognoscitivo que están asociadas con mecanismos mentales que demandan mayor procesamiento de la información, como lo es la prueba de Peabody, se asociaron con los niveles de zinc en eritrocitos, en una asociación altamente significativa $(p<0.012)^{68}$. Esta relación sugiere que la deficiencia de zinc en niños mexicanos puede afectar algunas áreas de su desarrollo cognoscitivo.

\section{Discusión}

Algunos estudios preliminares sugieren que la deficiencia moderada de zinc puede presentarse en una proporción elevada de los niños mexicanos. De manera similar a lo que ocurre con la deficiencia de hierro, la baja ingestión de zinc no parece ser el problema que más predispone a la deficiencia; la baja disponibilidad del zinc en la dieta rural mexicana constitutye la causa más probable de ese tipo de deficiencia. La dieta rural se compone en su mayoría de alimentos de origen vegetal, los cuales contienen potentes inhibidores de la absorción de zinc tales como el ácido fítico, la fibra dietética y el calcio. La presencia de estas sustancias y quizá de otras diminuye significativamente la biodisponibilidad del zinc.

Estudios de suplementación con zinc, doble ciego y utilizando placebo como control realizados en México, sugieren que la deficiencia moderada de zinc en niños mexicanos aumenta la presencia de enfermedades, particularmente de las diarreicas, y disminuye la capacidad de desarrollo cognoscitivo. Por el momento se desconoce la cantidad de niños que padecen esta deficiencia en diferentes regiones del país, lo cual hace necesarios más estudios al respecto; asimismo, se requiere encontrar los mecanismos mediante los cuales la deficencia de zinc afecta la morbilidad y otros aspectos de la salud humana.

\footnotetext{
* Sandstead, comunicación personal.
} 


\section{Referencias}

1. W illiams RJP. An introduction to the biochemistry of zinc. En: Mills DF, ed. Zinc in human biology. Berlin Heidelberg: Springer-Verlag, 1989: 15-31.

2. Cunningham BC, Bass $S$, Fuh $G$, W ells JA. Z inc medication of the binding of human growth hormone to the human prolactin receptor. Science 1990;250:1709-1712.

3. Bettger W J, O 'D ell BL.A critical physiological role of zinc in the structure and function of biomembranes. Life Sci 1981;28:1425-1438.

4. Hambidge KM, Casey CE, Krebs N F. Zinc. En: Mertz W, ed. Trace elements in human and animal nutrition. 5a edición. Florida:Academic Press, 1986;2:1-137.

5. Klug A, Rhodes D. Zinc fingers: A novel protein motif for nucleic acid recognition. Trends Biochem Sci 1987:464-469.

6. Hunt I, Murphy M, Martner P, Faraji B, Swendseid M, Reynolds R et al. Zinc, vitamin $B 6$, and other nutrients in pregnant women attending prenatal clinics in Mexico. Am J C lin N utr 1987;46:563-569.

7. Rosado JL, Bourges H, Saint-Martín B. Deficiencia de vitaminas y minerales en México. Una revisión crítica del estado de la información: I. Deficiencia de minerales. Salud Publica Mex 1995;37:130-139.

8. Jackson MJ, Jones DA, Edwards RHT, Swainbank IG, Coleman ML. Zinc homeostasis in man: Studies using a new stable isotope dilution technique. Br J N utr 1984;51:199-208.

9. Hambidge KM. Zinc and diarrhea. Acta Paediatr 1992;381 suppl:82-86. 10. Sandström B, Arvidsson B, Cederblad A, Björn-Rasmussen E. Zinc absorption from composite meals. I.The significance of wheat extraction rate, zinc, calcium, and protein content in meals based on bread. Am J C lin N utr 1980:33:739-745.

11. Madrigal H, Chávez A, Moreno-Terrazas 0, García T, Gutiérrez G. Consumo de alimentos y estado nutricional de la población del medio rural Mexicano. Rev Invest Clin 1986;38:9-19.

12. Sandström B, Lönnerdal B. Promoters and antagonists of zinc absorption. En: Mills C F , ed. Zinc in human biology (International Life Sciences Institute Human N utrition Reviews). Berlin: Springer Verlang, 1989:57-78. 13. Rosado JL, Díaz M. Propiedades fisicoquímicas relacionadas con la función gastrointestinal de seis fuentes de fibra dietética. Rev Inv Clin 1995; 47:283-289.

14.W ise A. D ietary factors determining the biological activities of phytate. Nutr Rev 1983:53:791-806.

15. Rosado JL, López P, Morales M, A llen LH . Fiber digestibility and breathhydrogen excretion in subjects consuming rural and urban Mexican diets. Am J Clin N utr 1991;53:55-60.

16. Rosado JL, López P, Morales M, Muñoz E, Allen LH. Bioavailability of energy, nitrogen, fat, zinc, iron and calcium from rural and urban Mexican diets. Br J N utr 1992;68:45-58.

17. Instituto $\mathrm{N}$ acional de la N utrición. Encuesta nacional de alimentación en el medio rural, 1989. Publicación L-86 México, D.F.: División de N utrición, 1990.

18. Prasad AS, Miale A, Farid Z, Sandstead HH, Schulert AR. Zinc metabolism in patients with syndrome of iron deficiency anemia, hepatosplenomegaly, dwarfism, and hypogonadism. J Lab C lin Med 1963;61:537-549. 19. Ronaghy HS, Spivey Fox MR, Garn SM, Israel H, Harp A, Moe PG et al. Controlled zinc supplementation for malnourished school boys pilot experiment. Am J C lin N utr 1963;22:1279-1289.

20. Ronaghy HS, Reinhold JG, Mahloudji M, Ghavami P, Spivey Fox MR, Halsted JA. Zinc supplementation of malnourished school boys in Iran: Increased growth and other effects. Am J C lin N utr 1974;27:112-121.

21. Hambidge KM, Hambidge $C$, Jacobs $M$, Baum JD. Low levels of zinc in hair, anorexia, poor growth, and hypogeusia in children. Pediatr Res 1972; 6:868-874.
22. W alravens PA, Hambidge KM. Growth of infants fed a zinc supplemented formula. Am J C lin N utr 1976;29:1114-1121.

23. Arcasoy A, Cavdar AO, Babacan E. Decreased iron and zinc absorption in Turkish children with iron deficiency and geophagia. Acta Hematol 1978;60:76-87.

24. Buzina R, Jusic M, Sapunar J, Milanovic N. Zinc nutrition and taste acuity in school children with impaired growth. Am J C lin N utr 1980;33: 2262-2267.

25.W alravens PA, Krebs N F, Hambidge KM. Linear growth of low income preschool children receiving a zinc supplement. Am J C lin N utr 1983;38: 195-201.

26. W alravens PA, Hambidge KM, Koepfer DM. Zinc supplementation in infants with a nutritional pattern of failure to thrive: a double-blind controlled study. Pediatrics 1983:83:532-538.

27. W alravens PA, Chakar A, Mokni R, D enise J, Lemonnier D. Zinc supplements in breastfed infants. Lancet 1992;340:683-685.

28. Smit-Vanderkooy PD, Gibson RS. Food consumption patterns of $\mathrm{Ca}$ nadian preschool children in relation to zinc and growth status. Am J C lin N utr 1987; 45:609-616.

29. Gibson RS, Smit-Vanderkool PD, MacD onald AC, Goldman A, Ryan BA, Berry M. A growth-limiting, mild zinc-deficiency syndrome in some Southen 0 ntario boys with low height percentiles. Am J C lin N utr 1989;49: 1266-1273.

30. Castillo-D urán C, Heresi G, Fisberg M, Uauy R. C ontrolled trial of zinc supplementation during recovery from malnutrition: Effects on growth and immune function. Am J C lin N utr 1989;45:602-608.

31. Schlesinger L, Arévalo M, Arredondo S, Díaz M, Lönnerdal B, Stekel A. Effect of a zinc-fortified formula on inmunocompetence and growth of malnourished infants. Am J Clin N utr 1992;56:491-498.

32. Khanum S,Alam AN ,Anwar I,A kbar-Ali M, Mujibur-Rahaman M. Effect of zinc supplementation on the dietary intake and weight gain of Bangladeshi children recovering from protein-energy malnutrition. Eur J Clin N utr 1988;42:709-714.

33. Roy SK, Tomkins AM, Haider R, Behrens RH, A kramuzzaman SM.The importance of zinc deficiency in stunting and morbidity. International Congress of N utrition XV; 1993;713:Abstract.

34. Rodríguez $A$, Venegas $G V$, Torres $S$. Zinc supplementation of infants fetal malnourished. En: Reunión de la Sociedad Latinoamericana de $0 \mathrm{n}$ cología Pediatrica VIII, 1991.

35. Carter JP, G rivetti LE, D avis JT, N asiff S, Mansour A, Mousa W A et al. Growth and sexual development of adolescent Egyptian village boys. Effects of zinc, iron, and placebo supplementation. Am J C lin N utr 1969;22: 59-78.

36. Udomkesmale E, Dhanamitta S, Sirisinba S, Charoenkiatkul S, Tuntipopipat S, Banjong 0 , et al. Effect of vitamin A and zinc supplementation on the nutriture of children in N ortheastThailand. Am J C lin N utr 1992;56: 50-57.

37. Bates CJ, Evans PH, Dardenne M, Prentice A, Lunn PG, N orthropClewes $C A$. et al. A trial of zinc supplementation in young rural $G$ ambian children. Br J N utr 1993;69:243-255.

38. Cavan KR, Gibson RS, Grazioso CF, Isalgue AM, Ruz M, Solomons N W. G rowth and body composition of periurban G uatemalan children in relation to zinc status:A zinc intervention trial. Am J C lin N utr 1993;57: 344-352.

39. Ruz M, Cavan KR, Bettger W J, Gibson RS. Erythrocytes, erythrocyte membranes, neutrophils and platelets as biopsy materials for the assessment of zinc status in humans. $\mathrm{Br} J \mathrm{~N}$ utr 1992;68:515-527.

40. Sazawal S, Black RE, Bhan MK, Bhandari N, Sinha A, Jalla S. Effect of zinc supplementation during acute diarrhea on duration and severity of the episode. A community based, double-blind controlled trial. N Engl J Med 1995;333:839-844. 
41. Rosado JL, López P, Muñoz E, Martínez H,Allen LH. Zinc supplementation reduced morbidity, but neither zinc nor iron supplementation affected growth or body composition of Mexican preschoolers. Am J Clin N utr 1997;65:13-19.

42. $\mathrm{Ninh} \mathrm{H}$. Zinc supplementation increases growth and circulating insuline-like growth factor I (IGF-I) in growth retarded Vietnamese children. Am J C lin N utr 1996;63:514-519.

43. Ruel MT, Rivera JA, Santizo MC, Lönnerdal B, Brown K. Impact of zinc supplementation on morbidity from diarrhea and respiratory infections among rural Guatemalan children. Pediatrics 1997;99:808-813.

44. Ruz M, Cavan KR, Bettger W J, Thompson IU, Berry M, Gibson RS. Development of a dietary model for the study of mild zinc deficiency in humans and evaluation of some biochemical and functional indices of zinc status. Am J Clin N utr 1991;53: 1295-1303.

45. 0 konofua FE,Amole FA, EmofurietaW 0 , U gwu N C. Zinc and copper concentrations in plasma of pregnant women in N igeria. Int J Gynecol 0 bstet 1989;29:19-23.

46. O konofua FE, Isinkaye A, O nwudiegwu U,A mole FA, Emofurieta W A, U gwu N C. Plasma zinc and copper in pregnant Nigerian women at term and their newborn babies. Int J Gynecol 0 bstet 1990;32:243-245.

47. Hurley, LS, Swenerton H. Congenital malformations resulting from zinc deficiency rats. Proc Soc Exp Biol Med 1966;123:692-696.

48. Hambidge KM, N eldner KH, W alravens PA. Zinc, acrodermatitis enteropathica, and congenital malformations. Lancet 975:577-578.

49. Swanson CA, King JC. Zinc and pregnancy outcome. Am J Clin N utr 1987:46:763- 771.

50. Jameson S. Effects of zinc deficiency in human reproduction. Acta Med Scandi 1976;593 suppl:5-89.

51. Cavdar AO, Babacan E, Arcasoy A. Effect of nutrition on serum zinc concentrations during pregnancy in Turkish women.Am J C lin N utr 1980; 33:542-544.

52. C avdar AO, Bahceci M, A kar N , Erten J,Yavuz H. Effect of zinc supplementation in a Turkish woman with two previous anencephalic infants. Gynecol 0 bstet Invest 1991;32:123-125.

53. Cherry FF, Bennett EA, Bazzano GS, Johnson LK, Fosmire GJ, Batson HK. Plasma zinc in hypertension/toxemia and other reproductive variables in adolescent pregnancy. Am J Clin N utr 1981;34:2367-2375.

54. McMichael AJ, Dreosti IE, Gibson GT, Hartshorne JM, Buckley RA, Colley DP.A prospective study of serial maternal serum zinc levels and pregnancy outcome. Early Hum D ev 1982;7:59-69.
55. Soltan MH, Jenkins DM. Maternal and fetal plasma zinc concentration and fetal abnormality. Br J O bstet Gynecol 1982;89:56-58.

56. Hunt IF, Murphy N J, Cleaver AE, Faraji B, Swendseid ME, Browdy BL et al.Zinc supplementation during pregnancy in low-income teenagers of Mexican descent: Effects on selected blood constituents and on progress and outcome of pregnancy. Am J C lin N utr 1985;42:815-828.

57. Breskin MW, Worthington-Roberts BS, Knopp RH, Brown Z, Plovie $B$, Mottet NK et al. First trimester serum zinc concentrations in human pregnancy. Am J Clin N utr 1983;38:943-953.

58. Mukherjee MD, Sandstead HH, Ratnaparkhi MV, Johnson LK, Milne $D B$, Stelling HP. Maternal zinc, iron, folic acid, and protein nutriture and outcome of human pregnancy. Am J C lin N utr 1984;40:496-507.

59. Cherry FF, Sandstead HH, Rojas P, Johnson LK, Batson HK, W ang X B. Adolescent pregnancy: association among body weight, zinc nutriture, and pregnancy outcome. Am J Clin N utr 1989;50:945-954.

60. Simmer K, Lort-Philips L, James C, Thompson RPH. A double-blind trial of zinc supplementation in pregnancy. Eur J C lin N utr 1991;45:139144.

61. G oldenberg RL,TumuraT, N eggersY, C o oper RL, Johnston KE, D uBard $M B$ et al.The effect of zinc supplementation on pregnancy outcome. JAMA 1995;274:463-468.

62. Rosado JL, Muñoz E, López P, Allen LH. Absorption of zinc sulfate, methionine and polyscorbate in the presence and absence of a plantbased rural Mexican diet. N utr Res 1993;13:1141-1151.

63. Brown K. Suplementación con zinc y crecimiento en niños: un meta análisis de estudios de intervención. D ieta y Salud 1995;4(1-2):1-7

64. Rivera J, Rosado JL, Brown K, Flores M, González-C ossío T, Rivera M. Effect of multiple micronutrient supplementation on the growth of young rural Mexican children. FASEB J 1996:A290.

65. Castillo-Durán C, Vial P, Vavy R. Trace mineral balance during acute diarrhoea. J Pediatr 1988;13:452-457.

66. Anónimo. Zinc and copper wastage during acute diarrhoea. N utr Rev 1990;48:19-22.

67. Sandstead HH. Zinc: Essentiality for brain development and function. N utr Rev 1985;43:129-137.

68. Golub M, Keen C, Gershwin M, Hendrickx A. Developmental zinc deficiency and behavior.J N utr 1995;125:2263S-2271S.

69. López P, Muñoz E, Allen LH, Alatorre J, Rosado JL. Cognitive performance and its relation to zinc and iron nutritional status of Mexican children. FASEB J 1997;11(3):A655. 